\title{
Impact of positive ZEB1 expression in patients with epithelial ovarian carcinoma as an oncologic outcome-predicting indicator
}

\author{
JUN SAKATA ${ }^{1}$, HIROAKI KAJIYAMA ${ }^{1}$, SHIRO SUZUKI ${ }^{1}$, FUMI UTSUMI $^{1}$, KAORU NIIMI $^{1}$, \\ RYUICHIRO SEKIYA ${ }^{1}$, KIYOSUMI SHIBATA ${ }^{1}$, TAKESHI SENGA ${ }^{2}$ and FUMITAKA KIKKAWA ${ }^{1}$ \\ ${ }^{1}$ Department of Obstetrics and Gynecology; ${ }^{2}$ Division of Tumor Biology, \\ Graduate School of Medicine, Nagoya University, Nagoya 466-8550, Japan
}

Received November 30, 2016; Accepted June 2, 2017

DOI: $10.3892 / \mathrm{ol} .2017 .6658$

\begin{abstract}
Several previous studies have revealed that the expression of zinc finger E-box binding homeobox 1 (ZEB1) in solid malignancies has an important significance on the clinical outcome of patients. However, the association between ZEB1 expression and survival in patients with epithelial ovarian carcinoma (EOC) remains unclear. The objective of the present study was to examine the extent of ZEB1 expression in EOC using immunohistochemical staining and investigate its association with patient outcome. A total of 40 patients with EOC initially treated with cytoreductive surgery and systematic chemotherapy were enrolled. ZEB1 expression was immunohistochemically categorized as negative, weak, moderate and strong according to the size of the staining area, and intensity. Subsequently, the associations between ZEB1 expression and recurrence/progression-free survival (RFS) rate were examined. The median age of patients in the current study was 54 years old (range, 22-72 years old). Among these patients, 15 (37.5\%) exhibited International Federation of Gynecology and Obstetrics stage I disease, and $10(25.0 \%), 13(32.5 \%)$, and $2(5 \%)$ had stage II, III, and IV disease, respectively. No patients with negative expression of ZEB1 experienced recurrence. In addition, ZEB1 expression was identified to be a significant predictor of a poorer RFS rate compared with negative expression (negative vs. weak, moderate and strong, $\mathrm{P}=0.0126$ ). Furthermore, multivariate analyses revealed that moderate and strong ZEB1 expression levels were significant prognostic indicators of a poorer RFS rate in patients with EOC (hazard ratio, 2.265; 95\% confidence interval, 1.072-8.021; $\mathrm{P}=0.0349)$. Confining analysis to patients with the clear-cell/mucinous histological type, those with
\end{abstract}

Correspondence to: Dr Hiroaki Kajiyama, Department of Obstetrics and Gynecology, Graduate School of Medicine, Nagoya University, Truma-cho 65, Showa-ku, Nagoya 466-8550, Japan E-mail: kajiyama@med.nagoya-u.ac.jp

Key words: epithelial ovarian carcinoma, zinc finger E-box binding homeobox 1, epithelial-mesenchymal transition, recurrence/ progression-free survival, immunohistochemical staining moderate/strong ZEB1 expression demonstrated a significantly poorer RFS rate $(\mathrm{P}=0.0025)$. Positive $\mathrm{ZEB} 1$ expression may be an indicator to predict unfavorable RFS in patients with EOC.

\section{Introduction}

Epithelial-mesenchymal transition (EMT) is a cornerstone phenomenon in which epithelial cells lose cell polarity and their cell-to-cell adhesion and acquire increased motility and invasive hallmarks to become mesenchymal-like cells (1). Cells undergoing EMT degrade the neighboring microenvironment, and migrate from the primary site to new frontier organs. Several families of transcriptional repressors, including zinc finger E-box binding homeobox 1 (ZEB1), Twist, SNAIL, and basic helix-loop-helix factors, have been identified as direct downregulators of E-cadherin transcription and representative inducers of EMT $(2,3)$. Among these molecules, ZEB1 is known as a member of the zinc-finger E-box binding homeobox (ZFH) family, and this molecule suppresses the expression of certain microRNAs, such as miR-183, miR-203, and miR-200 family members, which function as inhibitors of stem-like hallmarks as well as positive inducers of epithelial differentiation (4). Also, it has been reported to be a major transcriptional factor in cancer progression/ metastasis (5). In fact, earlier studies reported that ZEB1 promotes tumor invasiveness and metastasis and is correlated with a poorer clinical prognosis in patients with several solid cancers (6-8). According to the prior report from Siebzehnrubl et al (9), ZEB1 is an important marker of glioblastoma recurrence, including the capability of evading chemotherapy, suggesting that this molecule acts in both glioblastoma invasion and chemoresistance. In addition, silencing ZEB1 expression could significantly restore the chemosensitivity of docetaxel-resistant human lung adenocarcinoma cells as well as inhibit their migratory ability through reversing the mesenchymal phenotype (10).

Epithelial ovarian cancer (EOC) is the one of the most lethal cancers among the gynecologic malignancies worldwide, with more than 238,700 newly diagnosed cases and 151,900 reported deaths per year (11). In general, epithelial ovarian carcinoma (EOC) is a neoplasm originating from surface epithelial cells of the ovary, and it is called a silent killer because most patients with this disease are less 
symptomatic until the tumor has widely formed metastases in the peritoneal cavity, systematic lymph nodes, and distant parenchymal organs. Therefore, a number of EOC patients are frequently diagnosed when they enter an advanced stage (12). The majority of patients with EOC are categorized into four histological subtypes: High/low-grade serous, clear-cell, endometrioid, and mucinous carcinoma (13). In this context, EOC is biologically heterogeneous in nature, with different epidemiological and genetic backgrounds, molecular profiles, and behavioral responses toward chemotherapy and other treatments $(13,14)$, resulting in the difficulties in establishing unified, satisfactory treatments. Moreover, approximately three in four EOC patients show a favorable initial response to cytotoxic chemotherapy; however they gradually become chemoresistant, leading to recurrence and death. Correctively, intrinsic and/or acquired resistance to chemotherapeutic agents is the primary obstacle in the actual treatment of patients with EOC. The lack of strategies to cope with the biological complexity and change to being treatment-refractory is one of the main causes preventing improvement of the patient prognosis. Thus, it is crucial to develop more precise and effective therapies from a biological point of view.

These clinical and molecular backgrounds led us to hypothesize thatZEB1 plays a central role in cancer progression, and that positive ZEB1 expression may be a helpful indicator to predict an unfavorable clinical outcome in patients with EOC. In the present study, the need for a novel investigation of the possible correlation between immunostaining expression of ZEB1 and other clinicopathologic indicators and the oncologic outcome of EOC patients was proposed.

\section{Patients and methods}

Patients and immunohistochemical staining. The total of 40 ovarian carcinomas were categorized into the following pathological types: 11 serous, 18 clear cell, 8 endometrioid, and 3 mucinous carcinomas. As the histological types, we adopted the World Health Organization (WHO) classification criteria. The clinical stage was assigned according to the International Federation of Gynecology and Obstetrics (FIGO) staging system $(15,16)$.

Tissue samples of EOC were collected after obtaining informed consent from EOC patients who had been surgically treated at Nagoya University Hospital between 2001 and 2006. The present study was approved by the Ethics committee of Nagoya University (Approval no. 1234). Formalin-fixed, paraffin-embedded tissue sections were cut at a thickness of $4 \mu \mathrm{m}$.For heat-induced epitope retrieval,deparaffinized sections in $0.01 \mathrm{M}$ citrate buffer (Target Retrieval Solution pH 6.1; Dako Japan Co., Ltd., Tokyo, Japan) were heated three times at $90^{\circ} \mathrm{C}$ for 5 min using a microwave oven. Immunohistochemical staining was performed using the avidin-biotin immunoperoxidase technique with the Histofine SAB-PO kit (Nichirei, Tokyo, Japan) according to the manufacturer's protocol, and the experimental procedure was comprehensively described previously (3). Sections were incubated at $4^{\circ} \mathrm{C}$ for $12 \mathrm{~h}$ with primary antibody (anti-rabbit-ZEB1 polyclonal, at a 1:1,000 dilution; Cell Signaling Technology, Inc., Danvers, MA, USA). The sections were rinsed and incubated for $30 \mathrm{~min}$ with biotinylated anti-rabbit IgG antibody (second antibody). As a negative control, the primary antibody was replaced with normal rabbit IgG at an appropriate dilution.

Evaluation of immunohistochemical staining. For the evaluation of the results of immunohistochemical staining, 10 fields of each specimen were selected, and evaluated with both low- (x100) and high-power (x400) microscopy. Two investigators assessed the slides without knowledge of the clinicopathologic features and were blinded to each other's evaluation. The two investigators were in agreement on all the slides examined. Based on the ZEB1 immunostaining activity, a four-tiered semiquantitative score was assigned according to the intensity and area of stained cells as follows: For the evaluation of ZEB1 expression, the staining intensity was scored as 0 (negative), 1 (weak), 2 (medium), or 3 (strong). Percentage of staining the area was scored as $0(0 \%), 1(1-10 \%)$, $2(10-50 \%)$, and $3(51 \%<)$ relative to the total tumor area. The sum of the staining intensity and area scores was calculated as the final score (0-6) for ZEB1. Tumors with a final score of 0 , $1-2,3-4$, or 5-6 were classified as showing negative, weakly, moderately, and strongly positive expression, respectively.

Statistics. The distributions of clinicopathologic factors were statistically assessed using the Chi-square test or Fisher's exact test. The Cochran-Armitage test for trend was used to examine whether the frequency of recurrence was significantly different with each staining intensity. The recurrence/progression-free survival (RFS) was defined as the time interval between the date of surgery and date of the last follow-up or recurrence/ progression. The survival curves were compared employing the Log-rank test. Survival analysis was conducted using the Kaplan-Meier method. The prognostic significance of ZEB1 expression concerning other clinicopathologic variables was assessed using the multivariate Cox's proportional hazard's analysis. All statistical analyses were performed with JMP Pro Ver.10.0 (SAS Institute Japan). $\mathrm{P}<0.05$ was considered to indicate a statistically significant difference.

\section{Results}

Patients' characteristics. Patients' characteristics are detailed in Table I. The median (range) age was 52 (22-72) years. The distributions of the FIGO stage were $37.5 \%(15 / 40)$ stage I, $25.0 \%$ (10/40) stage II, 32.5\% (13/40) stage III, and $5.0 \%(2 / 40)$ stage IV, respectively. Of all patients, $38(95 \%)$ were postoperatively administered more than 3 cycles of chemotherapy. Two patients $(5.0 \%)$ did not undergo postoperative chemotherapy owing to their strong wishes or severe complications. A total of 6 patients had residual tumor at the initial surgery. The ZEB1 immunoreactivity was classified into the four scoring types as described in 'Patients and methods' (negative, weakly, moderately, and strongly positive expressions). Representative images of each histological feature are shown in Fig. 1.

In several cases, the immunoexpression of ZEB1 was found in the stroma as well as carcinoma tissues. Of the 40 carcinomas, negative, weakly, moderately, and strongly positive ZEB1 immunoexpressions were observed in 7 (17.5\%), 14 $(35.0 \%), 11(27.5 \%)$, and $8(20.0 \%)$ patients, respectively. 
Table I. Patients' characteristics.

\begin{tabular}{|c|c|c|}
\hline Characteristics & No. & $\%$ \\
\hline Total & 40 & \\
\hline \multicolumn{3}{|l|}{ Age } \\
\hline Median (range) & $54(22-72)$ & \\
\hline$\leq 50$ & 13 & 32.5 \\
\hline$>50$ & 27 & 32.5 \\
\hline \multicolumn{3}{|l|}{ FIGO stage } \\
\hline I & 15 & 37.5 \\
\hline II & 10 & 25.0 \\
\hline III & 13 & 32.5 \\
\hline IV & 2 & 5.0 \\
\hline \multicolumn{3}{|l|}{ Histological type } \\
\hline Serous & 11 & 27.5 \\
\hline Mucinous & 3 & 7.5 \\
\hline Endometrioid & 8 & 20.0 \\
\hline Clear-cell & 18 & 45.0 \\
\hline \multicolumn{3}{|l|}{ Surgery } \\
\hline Standard surgery + RPN & 19 & 47.5 \\
\hline Standard surgery + intestine resection & 4 & 10.0 \\
\hline Standard surgery $^{\mathrm{a}}$ & 13 & 32.5 \\
\hline Exploratory laparotomy & 4 & 10.0 \\
\hline \multicolumn{3}{|l|}{ Chemotherapy } \\
\hline Taxane plus platinum & 37 & 92.5 \\
\hline Conventinal platinum-based & 1 & 2.5 \\
\hline None & 2 & 5.0 \\
\hline \multicolumn{3}{|l|}{ Recurrence/progression } \\
\hline Yes & 19 & 47.5 \\
\hline No & 21 & 52.5 \\
\hline \multicolumn{3}{|l|}{ ZEB1 immunostaining classification } \\
\hline Negative & 7 & 17.5 \\
\hline Weakly positive & 14 & 35.0 \\
\hline Moderately positive & 11 & 27.5 \\
\hline Strongly positive & 8 & 20.0 \\
\hline
\end{tabular}

FIGO, International Federation of Gynecology and Obstetrics; RPN, retroperitoneal lymphadenectomy; aStandard surgery, including hysterectomy, bilateral salpingo-oophorectomy, with or without omentectomy.

Table II shows the association between ZEB1 expression and clinicopathologic parameters of primary EOC. Two categorized ZEB1 expressions (negative + weakly, moderately + strongly positive) were not correlated with any of the clinicopathologic parameters examined: Age, histological type, FIGO stage, and surgical procedure.

Oncologic outcome and the extent of ZEBI positivity. The median follow-up duration was 94.8, ranging from 3.8-202.0 months in the surviving patients. During this period, 19 patients $(47.5 \%)$ developed recurrence. The median time to recurrence was 10.8 months. The five-year RFS rate of all EOC patients was $52.5 \%$.

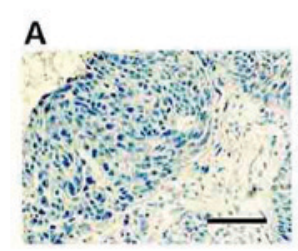

C

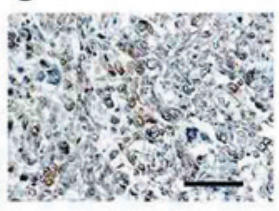

B

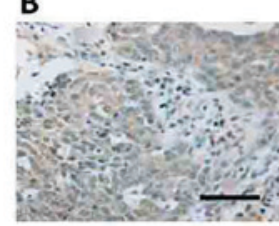

D

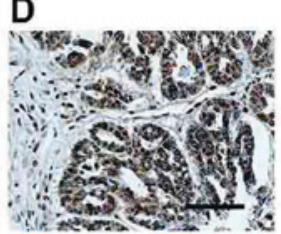

$\mathbf{E}$

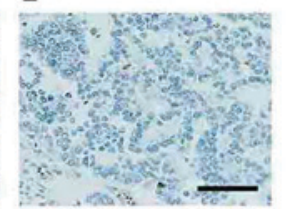

$\mathbf{F}$

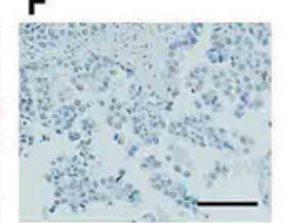

Figure 1.Survivalimpact ofZEB1 expressioninEOC tissues.Immunoreactivity of ZEB1 observed in surgical EOC samples (paraffin sections), positive or negative expression of ZEB1 in EOCs. (A) Negative (serous carcinoma), (B) weakly positive (clear-cell carcinoma), (C) moderately positive (serous carcinoma), (D) strongly positive (mucinous carcinoma); magnification x100. (E and F) Negative controls (the primary antibody was replaced with normal rabbit IgG: Clear-cell carcinoma), Bars: $100 \mu \mathrm{m}$.

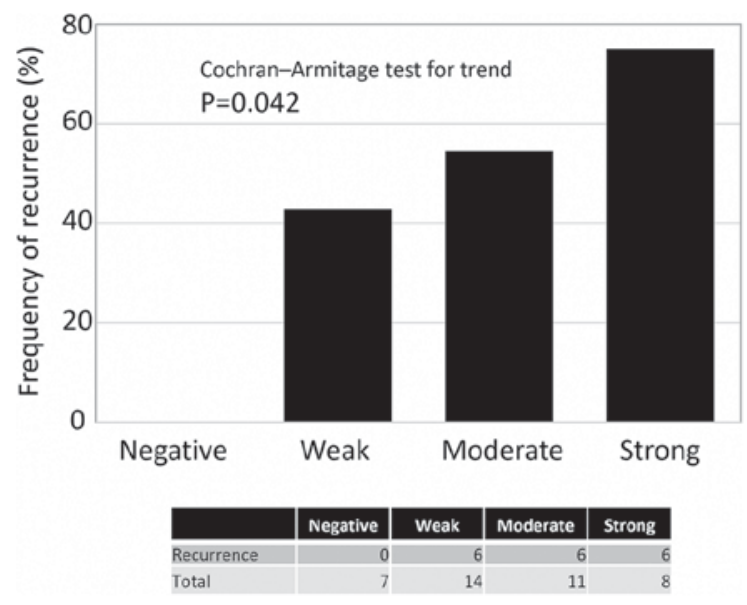

Figure 2. The frequency of recurrence according to the extent of ZEB1 positivity. There was no patient with negative expression of ZEB1 who experienced recurrence. Patients with higher ZEB1 positivity showed a higher rate of recurrence (Cochran-Armitage test for trend, $\mathrm{P}=0.042$ ).

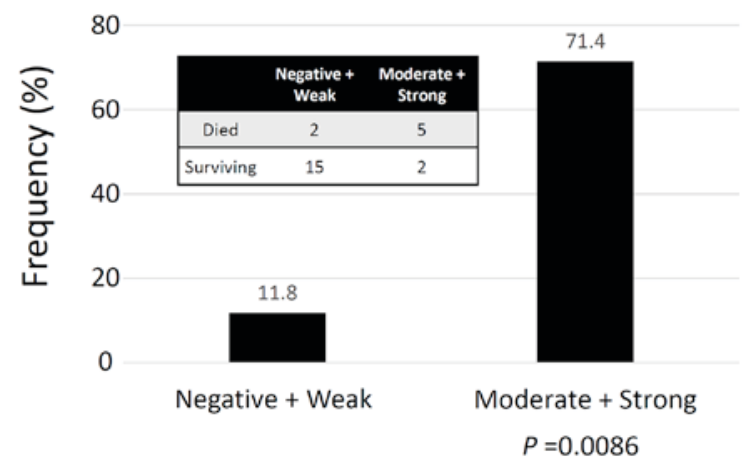

Figure 3. The frequency of recurrence according to the extent of ZEB1 positivity in patients with the clear-cell/mucinous histological type. Confining analysis to patients with the clear-cell/mucinous histological type, the frequency of an unfavorable oncologic outcome (death) was higher in patients with a higher ZEB1 positivity (Moderate + strong expression) (Fisher's exact test: $\mathrm{P}=0.0086$ ). 
Table II. Relationship between the expression of ZEB1 and clinicopathologic parameters of primary EOC.

\begin{tabular}{|c|c|c|c|c|c|}
\hline \multirow[b]{3}{*}{ Parameters } & \multicolumn{4}{|c|}{ ZEB1 } & \multirow[b]{3}{*}{ P-value } \\
\hline & \multicolumn{2}{|c|}{ Negative-Weak } & \multicolumn{2}{|c|}{ Moderate-Strong } & \\
\hline & $\mathrm{N}$ & $\%$ & $\mathrm{~N}$ & $\%$ & \\
\hline Total & 21 & 52.5 & 19 & 47.5 & \\
\hline \multicolumn{6}{|l|}{ Age } \\
\hline$\leq 50$ & 6 & 15.0 & 7 & 17.5 & \multirow[t]{2}{*}{0.737} \\
\hline$>50$ & 15 & 37.5 & 12 & 30 & \\
\hline \multicolumn{6}{|l|}{ FIGO } \\
\hline I+II & 15 & 37.5 & 10 & 25.0 & \multirow[t]{2}{*}{0.328} \\
\hline III+IV & 6 & 15.0 & 9 & 22.5 & \\
\hline \multicolumn{6}{|l|}{ Histological type } \\
\hline Serous & 4 & 10.0 & 7 & 17.5 & \multirow[t]{4}{*}{0.163} \\
\hline Mucinous & 3 & 7.5 & 0 & 0 & \\
\hline Endometrioid & 3 & 7.5 & 5 & 12.5 & \\
\hline Clear-cell & 11 & 27.5 & 7 & 17.5 & \\
\hline \multicolumn{6}{|l|}{ Surgery } \\
\hline Standard surgery + RPN & 11 & 27.5 & 8 & 20.0 & \multirow[t]{4}{*}{0.692} \\
\hline Standard surgery + intestine resection & 2 & 5 & 2 & 5.0 & \\
\hline Standard surgery ${ }^{\mathrm{a}}$ & 7 & 17.5 & 6 & 15.0 & \\
\hline Exploratory laparotomy & 1 & 2.5 & 3 & 7.5 & \\
\hline
\end{tabular}

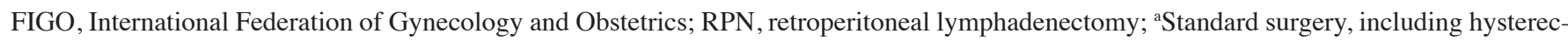
tomy, bilateral salpingo-oophorectomy, with or without omentectomy.
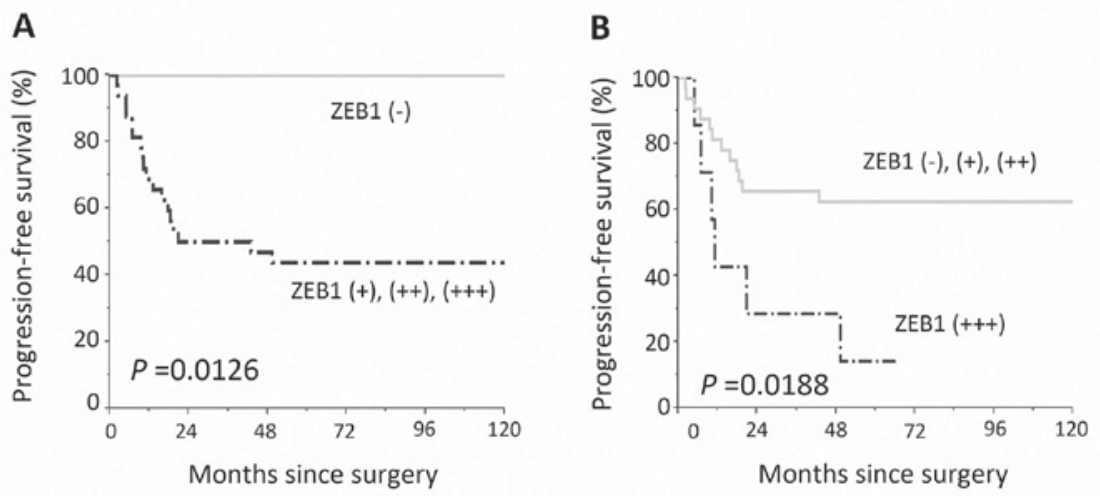

Figure 4. Kaplan-Meier progression-free survival curves for primary EOC patients according to the immunoreactivity of ZEB1. (A) Solid line indicates negative ZEB1 expression (negative: $\mathrm{N}=7$ ). The discontinuous line represents positive ZEB1 immunoexpression (weakly, moderately, and strongly positive: $\mathrm{N}=33$ ). Patients with positive ZEB1 expression showed a significantly poorer recurrence/progression-free survival (P=0.0126). (B) The solid line represents negative, weakly, and moderately positive $\mathrm{ZEB} 1$ expression $(\mathrm{N}=32)$. The discontinuous line represents strongly positive $\mathrm{ZEB} 1$ immunoexpression $(\mathrm{N}=8)$. Patients with strongly positive ZEB1 expression showed a significantly poorer recurrence/progression-free survival $(\mathrm{P}=0.0188)$.

Regarding the frequency of recurrence according to the extent of ZEB1 positivity, there was no patient with negative expression of ZEB1 who experienced recurrence. Patients with higher ZEB1 positivity showed a higher rate of recurrence (Cochran-Armitage test for trend, $\mathrm{P}=0.042$ ) (Fig. 2). Confining analysis to patients with the mucinous/clear-cell histological type, the frequency of an unfavorable oncologic outcome (death) was higher in patients with higher ZEB1 positivity (Moderate + Strong expression) $(\mathrm{P}=0.0086)$ (Fig. 3). Furthermore, compared with negative expression, two-scale positive ZEB1 expression predicted a significantly poorer RFS \{Negative vs. weak, moderate, and strong $(\mathrm{P}=0.002)$, Negative plus weak vs. moderate plus strong $(\mathrm{P}=0.001)\}$ (Fig. 4). Confining analysis to patients with the mucinous/clear-cell histological type, patients with lower ZEB1 expression (negative/weak) showed poorer RFS than those with higher ZEB1 expression (moderate/strong) $(\mathrm{P}=0.0025)$ (Fig. 5). In the current survival analyses, the post-hoc powers we calculated were ranging from 0.565 to 0.786 . 


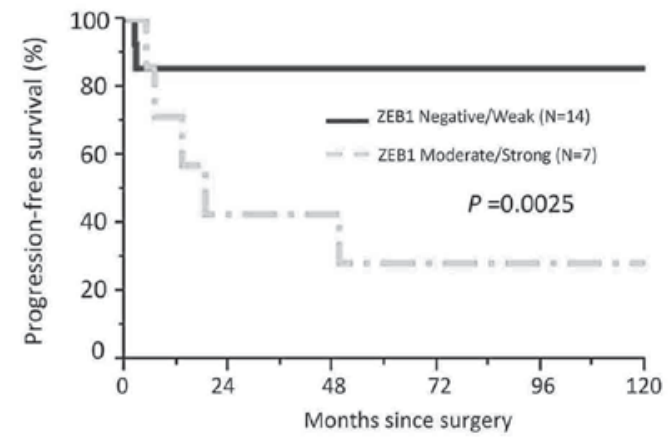

Figure 5. Kaplan-Meier progression-free survival curves in patients with the mucinous/clear-cell histological type according to the immunoexpression of ZEB1. The solid line represents negative-weakly positive ZEB1 expression (negative: $\mathrm{N}=14)$. The discontinuous line represents moderately-strongly positive ZEB1 immunoexpression ( $\mathrm{N}=7)$. Patients with moderate-strong ZEB1 expression showed a significantly poorer recurrence/progression-free survival $(\mathrm{P}=0.0025)$

Multivariate analysis. In multivariate RFS analyses, age ( $\leq 50$ vs. $>50)$, FIGO stage (I+II vs. III+IV), histological type (serous/endometrioid vs. mucinous/clear-cell), and ZEB1 immunoreactivity (negative/weak vs. moderate/strong) were included in the Cox proportional hazard analysis. The age, histological type, and ZEB1 expression were significant independent prognostic indicators of a poor RFS. The hazard ratio (HR) for moderately/strongly positive ZEB1 expression was as follows: HR: $2.2265,95 \%$ CI: 1.102-8.021; $\mathrm{P}=0.0349)$ (Table III).

Recurrence site in relapsed patients. Table IV shows the clinical features in the 18 relapsed patients. ZEB1 was expressed in 17 of the $18(94.4 \%)$ patients. In the majority of relapsed patients, the most frequent site of recurrence was the peritoneal cavity (16/18:88.9\%).

\section{Discussion}

In general, patients with EOC show an unfavorable prognosis, principally owing to its asymptomatic hallmark until the late stage, and it is frequently linked with disseminated intraperitoneal and/or distant metastases (17-19). Especially, peritoneal dissemination is the most common presentation, consisting of multi steps: First, tumor cells are released from the original tumor, and then they migrate in the abdominal cavity. When the tumor cells attach to the peritoneum, they start to invade tissues through the mesothelium $(20,21)$. In the current investigation of $40 \mathrm{EOC}$ patients, various levels of ZEB1 expression were identified in $82.5 \%$ (33 of 40), and patients with higher ZEB1 expressions showed a significantly poorer prognosis. Furthermore, multivariate analyses showed that a higher expression of ZEB1 was an independent prognostic indicator of a poorer RFS of EOC patients. Currently, studies demonstrated the important association between ZEB1 expression and aggressive phenotypes in several solid malignancies. Spoelstra et al (22) revealed that ZEB1 was aberrantly expressed in carcinoma cells of aggressive poorly-differentiated endometrioid carcinomas and other kinds of aggressive endometrial cancers, including uterine serous carcinomas. In addition, Hashiguchi et al (8) reported the clinical effect of
ZEB1 and E-cadherin expression in 108 patients with primary hepatocellular carcinoma. They demonstrated that positive immunohistochemical activity of ZEB1 was significantly correlated with a reduced expression of E-cadherin, and those with positive ZEB1/reduced E-cadherin expression particularly showed a poorer overall survival. These previous findings are consistent with our current results. If ZEB1 is directly linked with EMT of EOC, a ZEB1-positive clone may easily spread into the peritoneal cavity and have a greater opportunity to adhere to the mesothelium, resulting in the increasing formation of micro-and/or macroscopic peritoneal disseminations. According to earlier study from Chen et al (23), silencing of ZEB1 expression induced the colony-forming, wound-healing, and cellular migration abilities were downregulated with enhanced the expression of miR-200c to inhibit the epithelial-mesenchymal transition in ovarian cancer cells. In our next study, we aim to perform functional analysis of ZEB1 in EOC. At least, the current findings indicate that the immunoreactive identification of ZEB1 expression might be a crucial predictor of patients who will show a poor oncologic outcome and its identification may lead to the selection of better treatment strategies.

As well as metastasis to the peritoneal cavity and distant parenchymal organs, intrinsic or acquired chemoresistance remains a major challenge to improve the prognosis of patients with EOC. Recently, several studies suggested that the EMT shows therapy resistance, resulting in tumor recurrence (24-26). Also, the EMT-inducer ZEB1 was revealed to be involved in tumor stemness and treatment resistance. ZEB1 represses miR-200 as well as miR-203, which can also suppress stemness hallmarks (27). The level of ZEB1 is also upregulated in melanoma cells with acquired resistance and in biopsies from relapsed patients during treatment (28). Additionally, patients with a mucinous and clear-cell histology generally showed a very low response rate to platinum-based chemotherapy, leading to intrinsic chemoresistance $(29,30)$. Indeed, in patients with the mucinous/clear-cell histological type, the frequency of an unfavorable oncologic outcome was higher in those with higher ZEB1 positivity. ZEB1 expression may be involved in inheriting or the acquisition of EOC chemoresistance. The mechanism of patients with ZEB1 expression showing an unfavorable clinical outcome may be due to both the chemoresistant hallmark and metastasis-promoting effect of ZEB1 in the peritoneum via EMT. Indeed, in our study, the majority of patients with positive ZEB1 expression experienced recurrence $\{17 / 18$ (94.4\%)\}. The remaining chemoresistant clone, which is linked with ZEB1 expression, may be a cause of the high rate of recurrence. However, a functional analysis of ZEB1-expressing EOC cells was not carried out. Therefore, we can only hypothesize regarding the possibility of close linkage between chemoresistance and ZEB1 expression in EOC at present. We hope to test this hypothesis in the next study in order to clarify the EOC-specific biological hallmarks.

In the present study, there were several limitations, including a non-prospective, exploratory study, limited patient number, heterogeneous treatment modalities, and different follow-up periods. Especially, reflecting the small-scale patient number, our study did not have sufficient power. Nevertheless, we observed statistically significant difference at least in the two group comparison regarding ZEB1 
Table III. Uni- and multivariable analyses of clinicopathologic parameters in relation to recurrence/progression-free survival of patients

\begin{tabular}{|c|c|c|c|c|}
\hline \multirow[b]{3}{*}{ Parameters } & \multicolumn{4}{|c|}{ Recurrence/progression-free survival } \\
\hline & \multicolumn{2}{|c|}{ Univariable analysis } & \multicolumn{2}{|c|}{ Multivariable analysis } \\
\hline & Hazard ratio $(95 \% \mathrm{CI})$ & P-value & Hazard ratio $(95 \% \mathrm{CI})$ & P-value \\
\hline \multicolumn{5}{|l|}{ Age } \\
\hline$\leq 50$ & 1 & & 1 & \\
\hline$>50$ & $0.469(0.188-1.183)$ & 0.106 & $0.323(0.114-0.908)$ & 0.0325 \\
\hline \multicolumn{5}{|l|}{ FIGO } \\
\hline $\mathrm{I}-\mathrm{II}$ & 1 & & 1 & \\
\hline III-IV & $3.450(1.373-9.057)$ & 0.0087 & $5.013(1.679-15.952)$ & 0.0038 \\
\hline \multicolumn{5}{|l|}{ Histological type } \\
\hline $\mathrm{S} / \mathrm{E}$ & 1 & & 1 & \\
\hline $\mathrm{M} / \mathrm{C}$ & $0.529(0.202-1.320)$ & 0.172 & $0.738(0.422-3.211)$ & 0.2966 \\
\hline \multicolumn{5}{|l|}{ ZEB1 expression } \\
\hline Negative/weak & 1 & & 1 & \\
\hline Moderate/strong & 3.415 (1.329-9.865) & 0.010 & $2.265(1.072-8.021)$ & 0.0349 \\
\hline
\end{tabular}

FIGO, International Federation of Gynecology and Obstetrics; S/E, serous/endometrioid carcinoma; M/C, mucinous/clear-cell carcinoma.

Table IV. Clinical features and the ZEB1 immunostaining intensity in the relapsed patients.

\begin{tabular}{|c|c|c|c|c|c|c|c|}
\hline Case & Age & $\begin{array}{l}\text { Histological } \\
\text { type }\end{array}$ & $\begin{array}{l}\text { FIGO } \\
\text { stage }\end{array}$ & $\begin{array}{l}\text { Rec. } \\
\text { site }\end{array}$ & $\begin{array}{l}\text { Time to } \\
\text { Rec. (Mo) }\end{array}$ & $\begin{array}{l}\text { Follow-up } \\
\text { (Mo) }\end{array}$ & $\begin{array}{c}\text { ZEB1 } \\
\text { expression score }\end{array}$ \\
\hline 1 & 70 & S & IIIC & $\mathrm{PC}$ & 15.9 & 34.0 & 2 \\
\hline 2 & 50 & $\mathrm{~S}$ & IIC & PC, Distant & 43.4 & 107.3 & 3 \\
\hline 3 & 57 & S & IIIC & $\mathrm{PC}$ & 21.0 & 55.0 & 4 \\
\hline 4 & 61 & S & IVB & $\mathrm{PC}$ & 10.2 & 52.5 & 4 \\
\hline 5 & 69 & S & IIIC & $\mathrm{PC}$ & 10.4 & 38.8 & 3 \\
\hline 6 & 47 & S & IVB & $\mathrm{PC}$ & 17.9 & 32.4 & 2 \\
\hline 7 & 48 & S & IIIC & $\mathrm{PC}$ & 9.6 & 31.4 & 2 \\
\hline 8 & 22 & M & IIB & $\mathrm{PC}$ & 2.2 & 3.8 & 2 \\
\hline 9 & 49 & $\mathrm{E}$ & IIIC & $\mathrm{PC}$ & 11.2 & 31.3 & 4 \\
\hline 10 & 53 & $\mathrm{E}$ & IIIC & RPM, Distant & 19.6 & 38.6 & 2 \\
\hline 11 & 53 & E & IIC & RPM, Distant & 4.9 & 84.2 & 4 \\
\hline 12 & 58 & $\mathrm{E}$ & IIIC & $\mathrm{PC}$ & 6.9 & 14.4 & 4 \\
\hline 13 & 53 & $\mathrm{C}$ & IC & $\mathrm{PC}$ & 6.8 & 10.8 & 3 \\
\hline 14 & 38 & $\mathrm{C}$ & IIB & $\mathrm{PC}$ & 13.3 & 24.1 & 3 \\
\hline 15 & 39 & $\mathrm{C}$ & IA & $\mathrm{PC}$ & 18.6 & 49.7 & 3 \\
\hline 16 & 57 & $\mathrm{C}$ & IIB & PC, Distant & 2.4 & 4.3 & 2 \\
\hline 17 & 57 & $\mathrm{C}$ & IIIC & $\mathrm{PC}$ & 49.9 & 74.8 & 4 \\
\hline 18 & 48 & $\mathrm{C}$ & IIIC & $\mathrm{PC}$ & 4.9 & 88.2 & 3 \\
\hline
\end{tabular}

FIGO (2014), International Federation of Gynecology and Obstetrics; S, serous carcinoma; M, mucinous carcinoma; E, endometrioid carcinoma; C, clear-cell carcinoma; PC, peritoneal cavity; RTN, retroperitoneal lymph node; Distant, distant parenchymal organs; Rec, recurrence.

expression (negative/weak vs. moderate/strong). The result indicates our patients were not necessary insufficient to withdraw the conclusion that the ZEB1 expression was significant prognostic indicator of EOC. In addition, the heterogeneity of EOC is now the biggest challenge in all relevant studies. To investigate the effect of the ZEB1 expression in each 
histological type, we had categorized patients into the mucinous/clear-cell and other histological type. Nevertheless, we sincerely felt that the number of patients was so limited. Therefore, our finding that there was an association between ZEB1 expression and the unfavorable oncologic outcome of EOC patients is only weakly supported. We need to reanalyze and confirm the expression of ZEB1 in EOC samples in a larger patient population.

In conclusion, to our knowledge, this is the first study showing that the expression of ZEB1 was closely associated with a poor oncologic outcome of patients with EOC. The current findings may be based on the metastasis- and/or chemoresistant-promoting effects of ZEB1, although further investigation is needed to clarify the molecular mechanisms of ZEB1. In addition, at present, there are a number of problems, including the histological heterogeneity and lack of power. Nevertheless, our evidence provided sheds some light on the clinical and biological behavior of this malignancy. Although the current findings must be confirmed by other future studies, the expression of ZEB1 can be a helpful predictor factor for metastasis and/or relapse of EOC. We believe that this will help improve EOC treatment by adding criteria for the administration of systematic therapy in the future.

\section{Acknowledgements}

We sincerely thank Dr K. Tamakoshi (Nagoya University, School of Health Science), for his advice on statistical analyses as an expert statistician.

\section{References}

1. Larue L and Bellacosa A: Epithelial-mesenchymal transition in development and cancer: Role of phosphatidylinositol 3 kinase/AKT pathways. Oncogene 24: 7443-7454, 2005.

2. Kalluri R and Weinberg RA: The basics of epithelial-mesenchymal transition. J Clin Invest 119: 1420-1428, 2009.

3. Hosono S, Kajiyama H, Terauchi M, Shibata K, Ino K, Nawa A and Kikkawa F: Expression of Twist increases the risk for recurrence and for poor survival in epithelial ovarian carcinoma patients. Br J Cancer 96: 314-320, 2007.

4. Yang Y, Ahn YH, Chen Y, Tan X, Guo L, Gibbons DL, Ungewiss C, Peng DH, Liu X, Lin SH, et al: ZEB1 sensitizes lung adenocarcinoma to metastasis suppression by PI3K antagonism. J Clin Invest 124: 2696-2708, 2014.

5. Wu WS, Heinrichs S, Xu D, Garrison SP, Zambetti GP, Adams JM and Look AT: Slug antagonizes p53-mediated apoptosis of hematopoietic progenitors by repressing puma. Cell 123: 641-653, 2005.

6. Spaderna S, Schmalhofer O, Wahlbuhl M, Dimmler A, Bauer K, Sultan A, Hlubek F, Jung A, Strand D, Eger A, et al: The transcriptional repressor ZEB1 promotes metastasis and loss of cell polarity in cancer. Cancer Res 68: 537-544, 2008.

7. Sanchez-Tilló E, Liu Y, de Barrios O, Siles L, Fanlo L, Cuatrecasas M, Darling DS, Dean DC, Castells A and Postigo A: EMT-activating transcription factors in cancer: Beyond EMT and tumor invasiveness. Cell Mol Life Sci 69: 3429-3456, 2012.

8. Hashiguchi M, Ueno S, Sakoda M, Iino S, Hiwatashi K, Minami K, Ando K, Mataki Y, Maemura K, Shinchi H, et al: Clinical implication of ZEB-1 and E-cadherin expression in hepatocellular carcinoma (HCC). BMC Cancer 13: 572, 2013.

9. Siebzehnrubl FA, Silver DJ, Tugertimur B, Deleyrolle LP, Siebzehnrubl D, Sarkisian MR, Devers KG, Yachnis AT, Kupper MD, Neal D, et al: The ZEB1 pathway links glioblastoma initiation, invasion and chemoresistance. EMBO Mol Med 5 : 1196-1212, 2013

10. Ren J, Chen Y, Song H, Chen L and Wang R: Inhibition of ZEB1 reverses EMT and chemoresistance in docetaxel-resistant human lung adenocarcinoma cell line. J Cell Biochem 114: 1395-1403, 2013.
11. Siegel RL, Miller KD and Jemal A: Cancer statistics, 2016. CA Cancer J Clin 66: 7-30, 2016.

12. Holschneider $\mathrm{CH}$ and Berek JS: Ovarian cancer: Epidemiology, biology, and prognostic factors. Semin Surg Oncol 19: 3-10, 2000.

13. Prat J: New insights into ovarian cancer pathology. Ann Oncol 23 (Suppl 10): x111-x117, 2012.

14. Groen RS, Gershenson DM and Fader AN: Updates and emerging therapies for rare epithelial ovarian cancers: One size no longer fits all. Gynecol Oncol 136: 373-383, 2015.

15. Zeppernick F and Meinhold-Heerlein I: The new FIGO staging system for ovarian, fallopian tube, and primary peritoneal cancer. Arch Gynecol Obstet 290: 839-842, 2014.

16. Chen VW, Ruiz B, Killeen JL, Coté TR, Wu XC and Correa CN: Pathology and classification of ovarian tumors. Cancer 97 (10 Suppl): S2631-S2642, 2003.

17. Kajiyama H, Shibata K, Mizuno M, Umezu T, Suzuki S, Yamamoto E, Fujiwara S, Kawai M, Nagasaka T and Kikkawa F: Long-term clinical outcome of patients with recurrent epithelial ovarian carcinoma: Is it the same for each histological type? Int J Gynecol Cancer 22: 394-399, 2012.

18. Kikkawa F, Nawa A, Ino K, Shibata K, Kajiyama H and Nomura S: Advances in treatment of epithelial ovarian cancer. Nagoya J Med Sci 68: 19-26, 2006

19. Yoshikawa N, Kajiyama H, Mizuno M, Shibata K, Kawai M, Nagasaka T and Kikkawa F: Clinicopathologic features of epithelial ovarian carcinoma in younger vs. older patients: Analysis in Japanese women. J Gynecol Oncol 25: 118-123, 2014.

20. Kajiyama H, Shibata K, Terauchi M, Ino K, Nawa A and Kikkawa F: Involvement of SDF-1alpha/CXCR4 axis in the enhanced peritoneal metastasis of epithelial ovarian carcinoma. Int J Cancer 122: 91-99, 2008.

21. Terauchi M, Kajiyama H, Yamashita M, Kato M, Tsukamoto H, Umezu T, Hosono S, Yamamoto E, Shibata K, Ino K, et al: Possible involvement of TWIST in enhanced peritoneal metastasis of epithelial ovarian carcinoma. Clin Exp Metastasis 24: 329-339, 2007.

22. Spoelstra NS, Manning NG, Higashi Y, Darling D, Singh M, Shroyer KR, Broaddus RR, Horwitz KB and Richer JK: The transcription factor ZEB1 is aberrantly expressed in aggressive uterine cancers. Cancer Res 66: 3893-3902, 2006.

23. Chen D, Wang J, Zhang Y, Chen J, Yang C, Cao W, Zhang H, Liu Y and Dou J: Effect of down-regulated transcriptional repressor ZEB1 on the epithelial-mesenchymal transition of ovarian cancer cells. Int J Gynecol Cancer 23: 1357-1366, 2013.

24. Sahai E and Marshall CJ: Differing modes of tumour cell invasion have distinct requirements for Rho/ROCK signalling and extracellular proteolysis. Nat Cell Biol 5: 711-719, 2003.

25. De Wever O, Demetter P, Mareel M and Bracke M: Stromal myofibroblasts are drivers of invasive cancer growth. Int $\mathbf{J}$ Cancer 123: 2229-2238, 2008.

26. Cheng GZ, Chan J, Wang Q, Zhang W, Sun CD and Wang LH: Twist transcriptionally up-regulates AKT2 in breast cancer cells leading to increased migration, invasion, and resistance to paclitaxel. Cancer Res 67: 1979-1987, 2007.

27. Wellner U,Schubert J,Burk UC, Schmalhofer O,Zhu F, Sonntag A, Waldvogel B, Vannier C, Darling D, zur Hausen A, et al: The EMT-activator ZEB1 promotes tumorigenicity by repressing stemness-inhibiting microRNAs. Nat Cell Biol 11: 1487-1495, 2009.

28. Richard G, Dalle S, Monet MA, Ligier M, Boespflug A, Pommier RM, de la Fouchardière A, Perier-Muzet M, Depaepe L, Barnault R, et al: ZEB1-mediated melanoma cell plasticity enhances resistance to MAPK inhibitors. EMBO Mol Med 8: 1143-1161, 2016.

29. Sugiyama T, Kamura T, Kigawa J, Terakawa N, Kikuchi Y, Kita T, Suzuki M, Sato I and Taguchi K: Clinical characteristics of clear cell carcinoma of the ovary: A distinct histologic type with poor prognosis and resistance to platinum-based chemotherapy. Cancer 88: 2584-2589, 2000.

30. Shimada M, Kigawa J, Ohishi Y, Yasuda M, Suzuki M, Hiura M, Nishimura R, Tabata T, Sugiyama T and Kaku T: Clinicopathological characteristics of mucinous adenocarcinoma of the ovary. Gynecol Oncol 113: 331-334, 2009. 